\title{
Efectos de un programa alternativo de promoción de la alimentación saludable en comedor escolar
}

\author{
Effects of an alternative program to promote healthy eating in \\ school canteen
}

Nuria Rico-Sapena (https://orcid.org/0000-0002-1194-7852) ${ }^{1}$

Maria Eugenia Galiana-Sanchez (http://orcid.org/0000-0001-6977-7692) ${ }^{1}$

Josep Bernabeu-Mestre (http://orcid.org/0000-0002-5386-0173) ${ }^{1}$

Eva Maria Trescastro-López (http://orcid.org/0000-0001-8378-1612) ${ }^{1}$

Joaquín Moncho Vasallo (http://orcid.org/0000-0003-4383-2571) ${ }^{1}$
${ }^{1}$ Universitat d'Alacant. Carretera Sant Vicent

del Raspeig s/n, Sant Vicent del Raspeig. 03690

Alicante Espanha. nurisa72@hotmail.com

\begin{abstract}
The objective of this study was to determine the effects on school feeding habits in an educational intervention alternative to the conventional model of feeding promotion to a center with school canteens and ecological garden project. Cross-sectional and comparative descriptive observational study of 111 boys and girls between 9 and 12 years old, in the 2013-15 period. A school with alternative intervention was compared with another one that only included contents of the Infant and Primary Education curriculum, by means of evaluation of adherence to the mediterranean diet of the students with a validated Kidmed questionnaire. The intervention center presented greater adherence to the mediterranean diet than the control center. In the intervention center, the percentage of students with optimal diet $(64.52 \%)$ was higher than those who needed to improve their diet (33.87\%), contrary to what occurs in control center $(42.86 \%$ and $51.02 \%$ respectively). The students of the intervention center presented a higher percentage of affirmative answers in Kidmed test. The program of promotion of healthy alternative food of the intervention center could be effective for the acquisition of a diet of greater adherence to the mediterranean diet. Keywords Food and Nutrition Education, Health Promotion, Mediterranean Diet, Surveys and Questionnaires, Organic Foods.
\end{abstract}

Resumen El objetivo del estudio fue determinar los efectos sobre los hábitos alimentarios de escolares en una intervención educativa alternativa al modelo convencional de promoción de alimentación a un centro con comedor y proyecto de huerto ecológico. Estudio observacional descriptivo de corte transversal y comparativo de 111 niños $y$ niñas entre 9 y 12 años, en el periodo 2013-15. Se comparó un colegio con intervención alternativa con otro que únicamente incluía contenidos propios del currículo de Educación Infantil y Primaria, mediante evaluación de adherencia a la dieta mediterránea del alumnado con cuestionario validado Kidmed. El centro de intervención presentó mayor adherencia a la dieta mediterránea que el centro control. En el centro de intervención el porcentaje de alumnado con dieta óptima (64,52\%) fue mayor que los que necesitaban mejorar su dieta $(33,87 \%)$, mientras que en el centro fue inferior (42,86\% y 51,02\% respectivamente). Los escolares del centro de intervención presentaron mayor porcentaje de respuestas afirmativas en items Kidmed. El programa de promoción de alimentación saludable alternativo del centro de intervención podría ser efectivo para la adquisición de una dieta de mayor adherencia a la dieta mediterránea. Palabras clave Educación Alimentaria y Nutricional, Promoción de la Salud, Dieta Mediterránea, Encuestas y Cuestionarios, Alimentos Ecológicos. 


\section{Introducción}

La alimentación es uno de los aspectos más importantes que contribuyen a mantener la salud $y$, durante la edad escolar, resulta clave para que niños y niñas puedan tener un crecimiento adecuado $^{1,2}$. La dieta mediterránea (DM) se considera uno de los patrones alimentarios más saludables $^{3,4}$ para aquellos contextos culturales en los que sea posible llevarla a cabo, pese a que puede no ser adaptada a todos los países y realidades culturales $^{5}$. En los últimos años, la incorporación de la comida rápida y la globalización de la alimentación han provocado un alejamiento del patrón de la DM y una modificación de hábitos alimentarios que afecta especialmente a la población más joven ${ }^{1-3}$, contribuyendo a aumentar la prevalencia de problemas sanitarios como el sobrepeso y la obesidad infantil y juvenil ${ }^{1-6}$.

El aumento de la demanda del servicio de comedor escolar, impulsada por las nuevas realidades sociales, hace que las empresas de catering que sirven a estos comedores estén adquiriendo cada día más importancia ${ }^{7-9}$. El servicio de catering de los comedores escolares desempeña una importante función alimentaria, en cuanto al suministro de menús equilibrados, variados y saludables; nutricional, al satisfacer las necesidades específicas del alumnado; y educativa, ya que la comida que se sirve a diario es una herramienta útil para la adquisición de hábitos alimentarios saludables ${ }^{8-10}$.

Desde la Organización Mundial de la Salud se ha considerado necesario elaborar estrategias que favorezcan que la alimentación que se sirva en los colegios sea de acuerdo con los parámetros de una dieta saludable ${ }^{10,11}$. En este contexto la elección de estrategias educativas acordes con las necesidades y características de los escolares es fundamental para garantizar el logro de los objetivos educativos. Aspectos como la creación de entornos que favorezcan la alimentación saludable, el uso de métodos activos como las dinámicas de grupo, demostraciones y talleres, así como la elección de los soportes adecuados, favorecerán la motivación y participación de los escolares en las actividades educativas y el logro de mejoras nutricionales ${ }^{12}$.

Los comedores escolares, con el uso de alimentos ecológicos (aquellos que se obtienen sin el empleo de pesticidas, sustancias o aditivos químicos y en los que, para su producción se usan una serie de técnicas no contaminantes encaminadas a la protección del medio ambiente según el Reglamento Europeo No834/2007 del conse- jo del 28 de junio de 2007), pueden contribuir a abrir nuevas perspectivas en la restauración social, apoyando sistemas agroalimentarios sostenibles ${ }^{13,14}$. Proyectos como la implantación de huertos ecológicos escolares son un excelente recurso que posibilitan a un alumnado mayoritariamente urbano, experiencias con su entorno natural y rural, y permiten poner en práctica actitudes y hábitos de cuidado y responsabilidad medioambiental ${ }^{13}$. En el comedor escolar con proyecto de huerto ecológico, se descubre el valor de una buena alimentación y de calidad, así como la importancia de conocer el origen de lo que se come. Además, se fomenta el consumo de alimentos que se han sembrado en el huerto y se prueban nuevos sabores ${ }^{10,14}$.

Los comedores escolares que incorporan alimentos ecológicos y/o de proximidad son una oportunidad para promover un modelo de consumo y de producción local basado en 4 pilares fundamentales: 1- Salud, a través del fomento de una dieta equilibrada, basada en alimentos libres de productos químicos. 2- Educación, creando hábitos alimentarios saludables y sostenibles, respetuosos con el medio ambiente. 3- Desarrollo Rural, fomentando la producción ecológica de proximidad, impulsando la agrupación de pequeños productores y el desarrollo del medio rural. 4- Medio Ambiente, la producción agraria ecológica respeta el medio agrario y sus ciclos naturales, la biodiversidad y garantiza el bienestar animal $^{10}$.

El estudio de intervenciones educativas de promoción de la alimentación y nutrición y su comparación con otros centros en los que no se realizan programas específicos de promoción de la alimentación y nutrición más allá de las actividades contempladas en el propio currículo de Educación Infantil y Primaria, puede contribuir al conocimiento de los factores que influyen sobre los hábitos alimentarios de los escolares.

El objetivo principal del estudio fue determinar los efectos sobre los hábitos alimentarios de los escolares de una intervención educativa alternativa al modelo convencional de promoción de alimentación de un centro con comedor y proyecto de huerto ecológico.

\section{Métodos}

Estudio observacional descriptivo de corte transversal y comparativo. Se seleccionaron dos centros de Educación Infantil y Primaria pertenecientes a la Comarca del Bajo Segura de la 
provincia de Alicante (Comunidad Valenciana - España) con el mismo servicio de catering. Se consideró como grupo de intervención (CI) al alumnado de un centro escolar que, además de los contenidos sobre alimentación incluidos en el currículo de Educación Infantil y Primaria, contaba con un Plan de Promoción de la Alimentación Saludable. Además disponía de un comedor y huerto escolar ecológicos donde existía la posibilidad de incorporar los productos del propio huerto y otros de procedencia local en los menús diseñados por el catering. Este plan se basaba en cuatro aspectos fundamentales: el fomento de la dieta mediterránea, la comensalidad y los hábitos en la mesa, la introducción de alimentos ecológicos, y la importancia del consumo de agua. Las actividades se concretaron en: jornadas anuales de comida ecológica dirigidas a tutores/as y escolares, celebración del día mundial del agua y de la alimentación (anual), programa "Cinco frutas y cinco verduras al día” (niños/as exprimían y consumían zumo de naranja una vez a la semana), "Almuerzo diario con fruta" (sustituyendo la bollería industrial), "Bit alimentario" (cada día se trabajaban las propiedades de un alimento), y el proyecto "Los Mistos" específico de educación infantil, donde cada clase se representaba con una fruta y diariamente se trabajaba a partir de dibujos, carteles y murales temas alimentarios y nutricionales.

Paralelamente en el comedor se desarrollaron actividades para fomentar la correcta utilización de los cubiertos y servilleta, el lavado de manos antes y después de la comida, la postura correcta en la mesa, no elevar la voz, el lavado de dientes después de comer, así como pedir las cosas por favor y dar las gracias. El programa además se complementaba con actividades de educación ambiental con los proyectos "Educando en verde" y "La Cajita Verde" de cooperativa escolar con el huerto ecológico y venta de sus productos al comedor escolar; así como un programa de fomento del deporte y actividades al aire libre. El responsable del programa era el director y el equipo de dirección del centro educativo. Las actividades fueron realizadas por el profesorado y personal del comedor del centro así como personal externo experto. El centro pertenece a la Red de Ecoescuelas Europeas, Red de escuelas asociadas de la UNESCO, Red de centros de Calidad de la Generalitat Valenciana y tiene el Sello de Escuela Saludable y Escuela de Acción de Greenpeace. En la presente investigación se evaluó la adherencia a la DM de los escolares, el consumo familiar de alimentos ecológicos y si la dieta que ofertaba el comedor escolar se ajustaba a las recomendaciones nutricionales.

Como grupo control (CC) se seleccionó al alumnado de un segundo centro escolar de similares características perteneciente a la misma área de influencia que el CI, pero sin programa de promoción de la alimentación más allá de los contenidos propios del currículo de Educación Infantil y Primaria. Estos contenidos se imparten obligatoriamente en todos los centros educativos de la Comunidad Valenciana (también en el CI) en materias de Educación Infantil y de primer, segundo y tercer ciclo de Educación Primaria. En Educación Infantil (3-5 años), en el Bloque de cuidado personal y la salud, se incluye un tema sobre "Las acciones que favorecen la salud, la alimentación y el descanso". En primer ciclo (6-7 años), en el "Bloque de Ciencias, la salud y el desarrollo personal de la vida" incluye un tema sobre "los alimentos: su función en el organismo. Hábitos de alimentación saludable: la dieta equilibrada”. En segundo ciclo (8-9 años), en el "Bloque de Ciencias, la salud y el desarrollo personal" se incluye un tema sobre "Hábitos saludables (alimentación, higiene, ejercicio físico, descanso, utilización del tiempo libre). En tercer ciclo (10-11 años), en el "Bloque Ciencias, la salud y el desarrollo personal" se incluye tema sobre "Avances de la ciencia que mejoran la salud y la alimentación". Una vez seleccionados los centros, se mantuvieron reuniones informativas donde se procedió a explicar detalladamente el objeto del estudio, se les solicitó el programa educativo y del comedor y se programó de manera consensuada las diferentes fases del estudio, así como la entrega de documentación a tutores/as, necesaria para el estudio.

La intervención se inició cuando los alumnos accedieron a primer ciclo de Educación Infantil y Primaria con 3 años y se mantuvo activa hasta la finalización del estudio (2015).

La población del estudio la constituyeron niños y niñas con edades comprendidas entre los 9 y los 12 años, usuarios del comedor escolar de los centros escolares elegidos como CI y CC durante el periodo 2013-15.

Para la valoración de la adherencia a la DM se utilizó el cuestionario validado Kidmed ${ }^{15}$. Este cuestionario está compuesto por 16 ítems con enfoque dicotómico, estableciendo sólo dos alternativas de respuesta, "si" o "no", que se administró siguiendo las instrucciones de puntuación del estudio original. Así, las preguntas con connotaciones negativas respecto a la DM fueron valoradas como -1, y las que mostraron patrones 
asociados a la DM como +1. La puntuación total del Kidmed se sitúa, por tanto, entre los 0 y los 12 puntos. La cumplimentación del cuestionario se realizó en presencia de la investigadora con objeto de aclarar cualquier duda al alumnado.

A partir de la suma de los valores obtenidos en los 16 ítems se determinó el grado de adherencia a la DM estableciéndose tres niveles: KIDMED $\leq$ 3 (dieta de muy baja calidad); $4 \leq \mathrm{KIDMED} \leq 7$ (necesidad de mejorar el patrón alimentario para adecuarlo al modelo mediterráneo) y KIDMED $\geq 8$ (DM óptima).

La información proporcionada por el cuestionario Kidmed fue complementada con un cuestionario sociodemográfico y clínico de elaboración propia inspirado en los cuestionarios del Programa Perseo de la Estrategia NAOS ${ }^{16}$ (Estrategia para la Nutrición, Actividad Física y Prevención de la Obesidad), promovida por el Ministerio de Sanidad y Consumo, Ministerio de Educación y Ciencia y la Agencia Española de Seguridad Alimentaria y Nutrición (AESAN), que se entregaba el mismo día y que había sido rellenado por los tutores/as previamente. Este cuestionario incluía información sobre: variables sociodemográficas, clínicas y de estilos de vida.

También se solicitó al centro escolar el menú suministrado en el comedor para su valoración y para determinar si se ajustaba a las recomendaciones nutricionales y al patrón alimentario de la DM. Se solicitaron los menús de febrero y mayo, para disponer de un menú de invierno y otro de primavera. El análisis de menús se realizó mediante la valoración de la frecuencia de grupos de alimentos semanal según la tabla de frecuencias de consumos recomendadas en los menús de los centros escolares ${ }^{7}$.

La participación del alumnado fue voluntaria y en todo momento se garantizó la confidencialidad de la información. El estudio se llevó a cabo cumpliendo los principios éticos de la Declaración de Helsinki sobre investigaciones en seres humanos y los procedimientos utilizados fueron realizados tras la obtención del consentimiento informado en función de la legislación vigente (Ley 41/2002 de 14 de noviembre, básica reguladora de la autonomía del paciente y de derechos y obligaciones en materia de información y documentación clínica), de los tutores/as.

Se realizó un análisis descriptivo de los datos, calculándose las medidas habituales para las variables cuantitativas (medias y desviaciones típicas) y tablas de distribución de frecuencias y porcentajes para las variables cualitativas. Para el estudio de las asociaciones entre las puntuacio- nes de cada uno de los ítems del Kidmed y el centro (para cada sexo), así como para la valoración del Kidmed (dieta óptima, necesidad de mejora, baja calidad) se utilizó la prueba ji-cuadrado o la prueba exacta de Fisher en función del cumplimiento de los requisitos de aplicación correspondientes. Para la comparación de la puntuación global del Kidmed según centro, para cada sexo se utilizó la prueba $\mathrm{t}$ de student o la prueba $\mathrm{U}$ de Mann-Whitney. Finalmente se construyó un modelo de regresión lineal múltiple con variable dependiente la puntuación global de Kidmed y variables independientes: centro, edad del alumno/a, sexo del alumno/a, edad del tutor/a, país de nacimiento (nativo/extranjero), nivel de estudios del tutor/a, ingresos familiares y estado civil del tutor/a, que fueron recogidas con el cuestionario sociodemográfico clínico. Las variables fueron introducidas en el modelo de forma progresiva y analizando en cada paso los posibles efectos de interacción. El modelo final fue reajustado incluyendo sólo aquéllas variables que hubieran mostrado significación estadística.

Los resultados se analizaron estadísticamente mediante el paquete estadístico PASW IMB SPSS Statistics 18 Microsoft Co. NY, USA.

\section{Resultados}

El número total de escolares participantes fue de 111, de los cuales 62 pertenecían al CI y 49 al CC. El 40,54\% fueron niños y el 59,46\% niñas, con edades comprendidas entre 9 y 12 años, siendo la media de 10,76 con una desviación típica de 0,85 años.

\section{Valoración global del Kidmed}

La puntuación media del test Kidmed para el total de participantes fue de 7,60 $\pm 2,10$, siendo el valor mínimo de 3 y el máximo 12. El CI presentó una puntuación media significativamente mayor al CC, tanto en niños como en niñas (Tabla 1). Las niñas obtuvieron mayores puntuaciones globales del Kidmed que los niños, si bien las diferencias no fueron estadísticamente significativas.

$\mathrm{Al}$ analizar la valoración del Kidmed (Dieta óptima, necesidad de mejora, baja calidad) se observó que, para ambos sexos, un 64,52\% de los niños pertenecientes al CI seguían una dieta óptima por sólo el 42,86\% en el CC (Tabla 2). El análisis desagregado por sexo aumentó las diferencias entre los dos centros, sobre todo en niños, con porcentajes del $60 \%$ en el CI y el $26,67 \%$ en 
Tabla 1. Puntuaciones del Test Kidmed por centro y sexo

\begin{tabular}{lccccc}
\hline \multirow{2}{*}{$\begin{array}{c}\text { Puntuación } \\
\text { Test Kidmed }\end{array}$} & \multicolumn{2}{c}{ Centro intervención } & \multicolumn{2}{c}{ Centro control } & \multirow{2}{*}{ P } \\
\cline { 2 - 5 } & $\mathbf{N}(\%)$ & Media $\pm \mathbf{D E}$ & $\mathbf{N}(\%)$ & Media $\pm \mathbf{D E}$ & \\
\hline Ambos sexos & $62(55,86 \%)$ & $8,13 \pm 1,03$ & $49(44,14 \%)$ & $6,94 \pm 2,15$ & $0,003^{\mathrm{a}}$ \\
Niños & $30(48,39 \%)$ & $8,00 \pm 2,12$ & $15(30,61 \%)$ & $5,87 \pm 2,75$ & $0,026^{\mathrm{b}}$ \\
Niñas & $32(51,61 \%)$ & $8,25 \pm 1,76$ & $34(69,39 \%)$ & $7,41 \pm 1,65$ & $0,006^{\mathrm{a}}$ \\
\hline
\end{tabular}

a: Prueba T para la igualdad de medias

b: Prueba U de Mann-Whitney

Tabla 2. Frecuencia y porcentajes de valoración de respuestas del Test Kidmed por centro y sexo

\begin{tabular}{|c|c|c|c|c|c|}
\hline \multirow{2}{*}{\multicolumn{2}{|c|}{$\begin{array}{c}\text { Valoración Test Kidmed } \\
\mathrm{N}(\%)\end{array}$}} & \multirow{2}{*}{$\begin{array}{c}\text { Centro intervención } \\
\mathrm{N}(\%)\end{array}$} & \multirow{2}{*}{$\begin{array}{c}\text { Centro control } \\
\mathrm{N}(\%)\end{array}$} & \multirow{2}{*}{ Total } & \multirow{2}{*}{$\mathbf{p}$} \\
\hline & & & & & \\
\hline Ambos & Dieta óptima & $40(64,52 \%)$ & $21(42,86 \%)$ & $61(54,96 \%)$ & $0,044^{\mathrm{a}}$ \\
\hline \multirow[t]{2}{*}{ sexos } & $\begin{array}{l}\text { Dieta con necesidad de } \\
\text { mejora }\end{array}$ & $21(33,87 \%)$ & $25(51,02 \%)$ & $46(41,44 \%)$ & \\
\hline & Dieta baja calidad & $1(1,61 \%)$ & $3(6,12 \%)$ & $4(3,60 \%)$ & \\
\hline \multirow[t]{3}{*}{ Niños } & Dieta óptima & $18(60,00 \%)$ & $4(26,67 \%)$ & $22(48,89 \%)$ & $0,016^{\mathrm{a}}$ \\
\hline & $\begin{array}{l}\text { Dieta con necesidad de } \\
\text { mejora }\end{array}$ & $12(40,00 \%)$ & $8(53,33 \%)$ & $20(44,44 \%)$ & \\
\hline & Dieta baja calidad & $0(0,00 \%)$ & $3(20,00 \%)$ & $3(6,67 \%)$ & \\
\hline \multirow[t]{3}{*}{ Niñas } & Dieta óptima & $22(68,75 \%)$ & $17(50,00 \%)$ & $39(59,09 \%)$ & $0,102^{\mathrm{a}}$ \\
\hline & $\begin{array}{l}\text { Dieta con necesidad de } \\
\text { mejora }\end{array}$ & $9(28,13 \%)$ & $17(50,00 \%)$ & $26(39,39 \%)$ & \\
\hline & Dieta baja calidad & $1(3,12 \%)$ & $0(0,00 \%)$ & $1(1,52 \%)$ & \\
\hline
\end{tabular}

a: Prueba exacta de Fisher

el CC, cuya diferencia resultó estadísticamente significativa. En niñas el resultado fue similar pero la diferencia fue menor y no significativa, situándose en torno al $68,75 \%$ en el CI y el 50\% en el CC.

\section{Valoración de cada uno de los ítems del Kidmed}

El análisis de cada uno de los ítems según centro (intervención o control) para ambos sexos evidenció diferencias significativas en el consumo de verduras frescas o cocinadas (ítems 8 y 9 ), pasta o arroz (ítem 12), comida rápida (ítem 15) y golosinas y/o caramelos (ítem 16). El CI mostró, en general, mayores porcentajes de consumo en los ítems asociados positivamente con la DM que el CC $(87,1 \%$ vs $69,4 \%$ ítem 8 y $43,5 \%$ vs $22,4 \%$ ítem 9), mientras que el CC lo hizo con los asociados negativamente $(38,8 \%$ vs $12,9 \%$ ítem 15 y $42,9 \%$ vs 12,9\% ítem 16). Tan sólo en el consumo de pasta o arroz ( $71,4 \%$ vs $43,5 \%$ ítem
12) que se asocia positivamente con la DM el CC presentó mejores porcentajes que el CI (Tabla 3).

En niños, se encontraron diferencias significativas entre el CI y el CC en el consumo de lácteos en el desayuno ( $96,7 \%$ vs $73,3 \%$ ítem 2), segundo lácteo en el día (93,3\% vs $53,3 \%$ ítem 5), comida rápida ( $20 \%$ vs $73,33 \%$ ítem 15$)$ y golosinas y/o caramelos ( $10 \%$ vs $40 \%$ ítem 16 ). Además se observó una diferencia al borde de la significación estadística en el consumo de frutos $\operatorname{secos}(56,67 \%$ vs $26,67 \%$ ítem $13, p=0,057)$. El CI mostró, en general, mayores porcentajes de consumo en los ítems asociados positivamente con la DM (ítems 2, 5 y 13) que el CC, mientras que el CC lo hizo con los asociados negativamente (ítems 15 y 16).

En niñas se observaron diferencias significativas entre el CI y el CC en el consumo de verduras frescas o cocinadas $(96,88 \%$ vs $76,47 \%$ ítem 8 y $46,9 \%$ vs $17,6 \%$ ítem 9) y el consumo de golosinas y/o caramelos ( $15,63 \%$ vs $44,12 \%$ ítem 16). Se encontraron diferencias al borde de la 
significación estadística en el consumo de un segundo lácteo ( $59,4 \%$ vs $82.4 \%$ ítem $5, \mathrm{p}=0,057)$, fruta o zumo natural $(81,25 \%$ vs $97,06 \%$ ítem $6, \mathrm{p}=0,051)$ y comida rápida $(6,25 \%$ vs $23,53 \%$ ítem 15, $\mathrm{p}=0,084)$.

Tanto en niños ( $40,0 \%$ vs $73,33 \%$ ítem 12$)$ como en niñas ( $46,88 \%$ vs $70,59 \%$ ítem 12$)$ los porcentajes de consumo de pasta o arroz fueron significativamente superiores en el CC que en el CI (en niñas al borde de la significación, $\mathrm{p}=0,050$ ).

\section{Puntuaciones globales del Kidmed y características sociodemográficas y clínicas}

En la Tabla 4 se muestran los resultados del análisis bivariado (efectos no ajustados) y multivariante (efectos ajustados) con variable dependiente: puntuación global del Kidmed; y variables independientes: centro, edad del alumno/a, sexo del alumno/a, edad del tutor/a, país de nacimiento (nativo/extranjero), nivel de estudios del tutor/a, ingresos familiares y estado civil del tutor/a, del cuestionario sociodemográfico y clínico.

El análisis bivariado mostró que, de todas las variables explicativas utilizadas, tan sólo el cen-

Tabla 3. Porcentajes de respuestas afirmativas de los ítems del Test Kidmed por centro y sexo

\begin{tabular}{|c|c|c|c|c|c|c|c|c|c|}
\hline \multirow{3}{*}{$\begin{array}{ll} & \text { Items } \\
\text { Centros }\end{array}$} & \multicolumn{3}{|c|}{ Ambos Sexos } & \multicolumn{3}{|c|}{ Niños } & \multicolumn{3}{|c|}{ Niñas } \\
\hline & \multicolumn{2}{|c|}{$\%$ SI } & \multirow[b]{2}{*}{$\mathbf{p}$} & \multicolumn{2}{|c|}{$\%$ SI } & \multirow{2}{*}{$\mathbf{p}$} & \multicolumn{2}{|c|}{$\%$ SI } & \multirow{2}{*}{$\mathbf{p}$} \\
\hline & CI & $\mathrm{CC}$ & & CI & $\mathrm{CC}$ & & $\mathrm{CI}$ & $\mathrm{CC}$ & \\
\hline 1.No desayunas & 3,2 & 2,0 & $1,000^{\mathrm{a}}$ & 0 & 6,7 & $0,333^{a}$ & 6,3 & 0 & $0,231^{\mathrm{a}}$ \\
\hline 2.Desayunas un lácteo & 91,9 & 89,8 & $0,747^{a}$ & 96,7 & 73,3 & $0,036^{\mathrm{a}}$ & 87,5 & 97,1 & $0,190^{\mathrm{a}}$ \\
\hline 3.Desayunas un cereal o derivado & 69,4 & 69,4 & $0,997^{\mathrm{b}}$ & 73,3 & 73,3 & $1,000^{\mathrm{a}}$ & 65,6 & 67,6 & $0,862^{\mathrm{b}}$ \\
\hline $\begin{array}{l}\text { 4.Desayunas bollería industrial, } \\
\text { galletas o pastelitos }\end{array}$ & 35,5 & 34,7 & $0,931^{\mathrm{b}}$ & 20,0 & 40,0 & $0,174^{\mathrm{a}}$ & 50,0 & 32,4 & $0,145^{\mathrm{b}}$ \\
\hline $\begin{array}{l}\text { 5.Tomas un segundo lácteo en } \\
\text { el día }\end{array}$ & 75,8 & 73,5 & $0,778^{\mathrm{b}}$ & 93,3 & 53,3 & $0,003^{\mathrm{a}}$ & 59,4 & 82,4 & $0,057^{\mathrm{b}}$ \\
\hline $\begin{array}{l}\text { 6.Tomas una fruta o un zumo } \\
\text { natural todos los días }\end{array}$ & 83,9 & 93,9 & $0,104^{\mathrm{b}}$ & 86,7 & 86,7 & $1,000^{\mathrm{a}}$ & 81,25 & 97,06 & $0,051^{\mathrm{a}}$ \\
\hline $\begin{array}{l}\text { 7.Tomas una segunda pieza de } \\
\text { fruta todos los días }\end{array}$ & 66,1 & 53,1 & $0,162^{\mathrm{a}}$ & 63,3 & 46,7 & $0,286^{\mathrm{b}}$ & 68,8 & 55,9 & $0,281^{\mathrm{b}}$ \\
\hline $\begin{array}{l}\text { 8.Tomas verduras frescas o } \\
\text { cocinadas una vez al día }\end{array}$ & 87,1 & 69,4 & $0,022^{\mathrm{b}}$ & 76,7 & 53,3 & $0,172^{\mathrm{a}}$ & 96,88 & 76,47 & $0,028^{\mathrm{a}}$ \\
\hline $\begin{array}{l}\text { 9.Tomas verduras frescas o } \\
\text { cocinadas más de una vez al día }\end{array}$ & 43,5 & 22,4 & $0,020^{\mathrm{b}}$ & 40 & 33,3 & $0,664^{\mathrm{b}}$ & 46,9 & 17,6 & $0,011^{\mathrm{b}}$ \\
\hline $\begin{array}{l}\text { 10.Tomas pescado con } \\
\text { regularidad (al menos } 2-3 \text { veces a } \\
\text { la semana) }\end{array}$ & 79,0 & 83,7 & $0,535^{\mathrm{b}}$ & 83,3 & 86,7 & $1,000^{\mathrm{a}}$ & 75,00 & 82,4 & $0,465^{\mathrm{b}}$ \\
\hline $\begin{array}{l}\text { 11.Tomas legumbres más de } 1 \mathrm{vez} \\
\text { a la semana }\end{array}$ & 91,9 & 81,6 & $0,104^{\mathrm{b}}$ & 86,7 & 73,3 & $0,410^{\mathrm{a}}$ & 96,9 & 85,3 & $0,198^{\mathrm{a}}$ \\
\hline $\begin{array}{l}\text { 12. Tomas pasta o arroz } 5 \text { días o } \\
\text { más a la semana }\end{array}$ & 43,5 & 71,4 & $0,003^{\mathrm{b}}$ & 40,00 & 73,33 & $0,035^{\mathrm{b}}$ & 46,88 & 70,59 & $0,050^{\mathrm{b}}$ \\
\hline $\begin{array}{l}\text { 13. Tomas frutos secos al menos } \\
2-3 \text { veces a la semana }\end{array}$ & 61,3 & 61,2 & $0,994^{\mathrm{b}}$ & 56,67 & 26,67 & $0,057^{\mathrm{b}}$ & 65,6 & 76,5 & $0,331^{\mathrm{b}}$ \\
\hline $\begin{array}{l}\text { 14.Se utiliza aceite de oliva en tu } \\
\text { casa }\end{array}$ & 100 & 98,0 & $0,441^{\mathrm{a}}$ & 100,00 & 93,3 & $0,333^{\mathrm{a}}$ & 100 & 100 & $1,000^{\mathrm{a}}$ \\
\hline $\begin{array}{l}\text { 15.Acudes una vez o más a la } \\
\text { semana a un centro de comida } \\
\text { rápida }\end{array}$ & 12,9 & 38,8 & $0,002^{\mathrm{b}}$ & 20,00 & 73,33 & $0,001^{\mathrm{b}}$ & 6,25 & 23,53 & $0,084^{\mathrm{a}}$ \\
\hline $\begin{array}{l}\text { 16. Tomas golosinas y/o caramelos } \\
\text { varias veces al día }\end{array}$ & 12,9 & 42,9 & $<0,001^{\mathrm{b}}$ & 10,00 & 40,00 & $0,042^{\mathrm{a}}$ & 15,63 & 44,12 & $0,012^{\mathrm{b}}$ \\
\hline
\end{tabular}


Tabla 4. Análisis multivariante para la puntuación global del Test Kidmed

\begin{tabular}{|c|c|c|c|c|c|c|c|c|c|}
\hline \multirow{3}{*}{\multicolumn{2}{|c|}{$\begin{array}{c}\text { Parámetros } \\
\text { B }\end{array}$}} & \multicolumn{4}{|c|}{ Efectos Crudos } & \multicolumn{4}{|c|}{ Efectos Ajustados } \\
\hline & & \multicolumn{2}{|c|}{ IC95\% } & \multirow{2}{*}{$\mathbf{p}$} & \multirow{2}{*}{ B } & \multicolumn{2}{|c|}{ IC95\% } & \multirow[b]{2}{*}{$\mathbf{p}$} & \\
\hline & & Inferior & Superior & & & Inferior & Superior & & \\
\hline \multirow[t]{2}{*}{ Centro } & Control & $-1,190$ & $-1,943$ & $-0,438$ & $0,002^{\mathrm{a}}$ & $-10,526$ & $-19,874$ & $-1,177$ & $0,027^{* * b}$ \\
\hline & Intervención & & & & & & & & \\
\hline \multirow[t]{2}{*}{ Sexo } & Niña & 0,529 & $-0,258$ & 1,316 & $0,188^{\mathrm{a}}$ & 0,849 & 0,097 & 1,602 & $0,027^{* \times b}$ \\
\hline & Niño & & & & & & & & \\
\hline \multirow[t]{2}{*}{ Origen } & Extranjero & 0,340 & $-0,878$ & 1,559 & $0,584^{\mathrm{a}}$ & & & & \\
\hline & Nativo & & & & & & & & \\
\hline \multirow{4}{*}{$\begin{array}{l}\text { Ingresos } \\
\text { familiares }\end{array}$} & $2701+$ & 0,978 & $-0,811$ & 2,766 & $0,713^{\mathrm{a}}$ & & & & \\
\hline & $1801-2700$ & $-0,072$ & $-2,052$ & 1,908 & & & & & \\
\hline & $901-1800$ & 0,278 & $-0,617$ & 1,172 & & & & & \\
\hline & $<=900$ & & & & & & & & \\
\hline \multirow{4}{*}{$\begin{array}{l}\text { Nivel de } \\
\text { estudios } \\
\text { tutores }\end{array}$} & Universitarios & 1 & 0,015 & 2,435 & $0,108^{\mathrm{a}}$ & & & & \\
\hline & Secundarios & 1 & $-0,279$ & 1,395 & & & & & \\
\hline & Primarios & & & & & & & & \\
\hline & / Sin estudios & & & & & & & & \\
\hline \multicolumn{2}{|c|}{ Edad alumno } & $-0,145$ & $-0,606$ & 0,315 & $0,563^{a}$ & $-1,355$ & $-1,078$ & 0,075 & $0,088^{\star b}$ \\
\hline \multicolumn{2}{|c|}{ Edad del tutor } & $-0,024$ & $-0,112$ & 0,063 & $0,587^{\mathrm{a}}$ & & & & \\
\hline \multirow{5}{*}{$\begin{array}{l}\text { Estado } \\
\text { civil } \\
\text { tutores }\end{array}$} & Pareja de hecho & 0,045 & $-3,745$ & 3,834 & $0,825^{\mathrm{a}}$ & & & & \\
\hline & Soltero & $-0,705$ & $-2,641$ & 1,231 & & & & & \\
\hline & Divorciado & $-0,336$ & $-1,277$ & 0,605 & & & & & \\
\hline & / Separado & & & & & & & & \\
\hline & Casado & & & & & & & & \\
\hline \multicolumn{2}{|c|}{ Interacción: Centro ${ }^{\star}$ Edad } & & & & & 0,854 & $-0,011$ & 1,718 & $0,053^{* \mathrm{~b}}$ \\
\hline
\end{tabular}

tro (intervención/control) presentó una relación significativa con la puntuación global del Kidmed (ver columnas de efectos no ajustados de la Tabla 4), de forma que el CC puntuaba 1,190 (IC95\%=[-1,943;-0,438]) puntos menos en el Kidmed que el CI.

El modelo de regresión lineal múltiple resultante de la introducción progresiva de las variables independientes mencionadas anteriormente incluyó finalmente, el centro, el sexo del niño/a y la edad. Además, los resultados apuntaban un posible efecto de interacción entre la edad y el centro $(\mathrm{p}=0,053)$ que se incluyó en el modelo final. Así, y ajustando por el resto de variables, se estimó que las niñas puntuarían 0,849 puntos más en el Kidmed que los niños (IC95\%=[0,097;1,602]). El CI puntuó, globalmente, más que el CC en el Kidmed y para todas las edades. Sin embargo, el efecto de interacción detectado apuntaría a que estas diferencias de puntuación serían mayores a edades tempranas y se atenuarían a medida que aumenta la edad del escolar y, con ello, la exposi- ción al programa de intervención. Así, para niños y niñas del mismo sexo, en el CI se produciría una disminución de la puntuación del Kidmed del niño/a de 0,501 por cada año más, mientras que en el CC se produciría un aumento de 0,353 puntos por cada año más.

\section{Consumo de alimentos ecológicos}

El análisis de la información sobre el consumo de alimentos ecológicos contenida en el cuestionario sociodemográfico y clínico permitió comprobar que el 60,2\% del alumnado participante en el estudio consumían productos mixtos (convencionales y ecológicos) y el resto exclusivamente convencionales. Se observaron diferencias por centros aunque éstas no fueron estadísticamente significativas. Así, mientras que en el CC el 50\% del alumnado consumía productos mixtos, este porcentaje ascendía al $67,3 \%$ en el caso del CI. 


\section{Adecuación de los menús a las recomendaciones nutricionales}

La empresa de catering que suministraba los alimentos fue la misma para los dos centros, de forma que los menús resultaron muy similares y pudo comprobarse que ambos cumplían las recomendaciones de frecuencia de consumo de alimentos.

Sin embargo, en la elaboración de los menús del CI se utilizaron alimentos ecológicos. Concretamente, pudo comprobarse que el $100 \%$ de las frutas eran ecológicas y un elevado porcentaje de verduras suministradas a los escolares procedían del huerto escolar ecológico. Además, se incluían verduras y frutas de temporada y de procedencia local. Por último, se apreció que en los menús del CI se utilizaba con mayor frecuencia la merluza como pescado elección mientras que en el CC fue la panga.

\section{Discusión}

El Plan de Promoción de la Alimentación Saludable llevado a cabo en el centro de intervención pone de manifiesto que la elección de estrategias educativas novedosas, en consonancia con otras experiencias tanto en el ámbito nacional como internacional ${ }^{11,12,17}$, y acordes con las necesidades de los escolares es fundamental para garantizar el logro de los objetivos educativos.

El CI obtuvo una puntuación del Kidmed significativamente superior al CC, tanto a nivel global como desagregado por sexos. Los datos obtenidos del test Kidmed mostraron que entre los escolares de los dos centros predominó una dieta de media a alta adherencia a la DM. Solo un 3,6\% del alumnado presentó una dieta de baja adherencia. El CI presentó un porcentaje mayor de escolares con dieta óptima que con dieta con necesidad de mejora y de baja calidad. Además, estos resultados fueron mejores que los del alumnado del CC, que presentaron un porcentaje mayor de estudiantes con una dieta de media adherencia y de dieta de baja calidad, siendo esta diferencia significativa. Esto indicaría que el programa educativo de promoción de la alimentación y nutrición realizado en el CI podría ser efectivo en la adquisición de hábitos alimentarios compatibles con la DM, al igual que otros programas de educación alimentaria ${ }^{6,17-19}$.

Otros estudios de ámbito nacional, pero con rangos de edad diferentes, también han mostrado resultados similares ${ }^{6,19-21}$, aunque parece que existe una tendencia por la cual, a mayor edad, hay menor adherencia a la $\mathrm{DM}^{6,15,20-26}$. En este estudio, también se aprecia esta tendencia progresiva de alejamiento de la adherencia a la DM según aumenta la edad de los escolares. Es decir, que los buenos hábitos alimentarios que definen la DM, se van perdiendo a medida que los estudiantes van creciendo y adoptando más autonomía e independencia a la hora de alimentarse en la edad adolescente.

Si bien los resultados obtenidos evidencian que los escolares del CI siguieron una tendencia de mayor deterioro de la adherencia en los grupos de edad más avanzados, en el CC ocurrió lo contrario. Así, según aumentaba la edad el alumnado, éstos iban adquiriendo hábitos más saludables y un acercamiento en su dieta a los patrones de la DM, aunque siempre por debajo de los niveles de adherencia del CI. Posiblemente la exposición a las diferentes actividades que se realizaban dentro del currículo de EP y en el comedor escolar facilitaban la adquisición de buenos hábitos alimentarios en el alumnado del CC. Por el contrario, en el CI podría suceder que una excesiva exposición en tiempo e intensidad del programa educativo alternativo de promoción a la alimentación y nutrición, pierda efecto o impacto en los escolares. Habría que evaluar la adecuada exposición de los programas de intervención para que sean lo más efectivos y perdurables posibles.

Las niñas presentaron un porcentaje mayor de dieta óptima que los niños en todos los casos, siendo esta diferencia significativa. Esto coincide con los resultados obtenidos en una investigación llevada a cabo con estudiantes universitario ${ }^{27}$. Sin embargo, en niños de menor edad, similar a la muestra de este estudio, se obtuvieron resultados diferentes, es decir, los niños mostraban una dieta de adherencia superior a las niñas respecto a los valores obtenidos en el test Kidmed ${ }^{18,22,24-26}$. Puede que niños y niñas estén influenciados de diferente manera por los estereotipos de culto al cuerpo o cuidado de la imagen que marca la sociedad ${ }^{28}$.

Evaluando independientemente las respuestas obtenidas de los diferentes ítems del Kidmed se obtuvieron algunas diferencias significativas entre los centros en lo relativo al consumo de verdura, pasta o arroz, lácteos, comida rápida y golosinas o caramelos, evidenciándose que los niños y niñas del CI, tenían unos hábitos alimentarios más cercanos a la DM que los niños y niñas del CC.

Entre los aspectos positivos de la dieta, cabe señalar que la mayoría de los escolares decía desayunar habitualmente, preferiblemente un lác- 
teo y cereales o derivados, declaran la utilización culinaria del aceite de oliva, el consumo diario de una ración de fruta y verduras y legumbres más de una vez a la semana. Por el contrario, se obtuvieron porcentajes relativamente importantes para preguntas con connotaciones negativas y que suponen un alejamiento de la DM, como son el consumo de bollería industrial, y en el caso del centro control, acudir semanalmente a restaurantes de comida rápida, y tomar golosinas o caramelos varias veces al día. Uno de los problemas de la sociedad actual y en concreto de los jóvenes es la gran accesibilidad a distintos tipos de alimentos, algunos ricos en nutrientes y una gran parte caracterizados por ser calorías vacías, que junto con la influencia de los medios de comunicación promueven una tendencia hacia el consumo de dietas desequilibradas y un abuso de comida rápida. En otros estudios se han obtenido resultados similares respecto al consumo de comida rápida, bollería industrial y golosinas, y caramelos $^{22-24,27}$. Se comprueba, por tanto, la necesidad de seguir incidiendo en evitar conductas alejadas a la DM y relegar estos alimentos a un consumo ocasional o esporádico.

El alumnado del CI consumió más alimentos ecológicos que el del CC, lo que podría explicarse por la estrategia de uso de alimentos ecológicos en el comedor escolar y por las intervenciones educativas recibidas. También habría que tener en cuenta que ambos centros se encuentran en poblaciones de origen agrícola, donde hay mayor facilidad de la adquisición de productos ecológicos, y productos locales en mercados rurales, incluso de familiares. Por otra parte, los productos ecológicos son más caros que los convencionales y ello podría ser un obstáculo para incorporarlos a la dieta diaria en sus respectivos hogares, impidiendo la detección de una diferencia mayor entre los dos centros que pudiera ser estadísticamente significativa para la muestra observada. De hecho, como ya se ha indicado, aunque el análisis de la dieta suministrada por la empresa de catering en el comedor escolar de ambos centros cumple las recomendaciones nutricionales para escolares establecidas por la Consejería de Sanidad de la Generalitat Valenciana, la principal diferencia es que, en los menús del CI, se utilizaron alimentos ecológicos, en concreto, el 100\% de las frutas suministradas a los escolares, y la muchas de las verduras consumidas y producidas por el propio huerto ecológico. También en el CI se utilizaba con mayor frecuencia la merluza como pescado elección mientras que en el CC fue la panga.
Entre las limitaciones más importantes del estudio cabe destacar que no ha sido posible realizar una evaluación inicial y continuada del programa que permitiera evaluar los cambios en los hábitos alimenticios del alumnado. Para paliar esta limitación, se eligió como centro educativo control uno de las mismas características (tamaño, carácter público) y que perteneciera a la misma área de influencia poblacional al que pertenecía el grupo de intervención. Además se comprobó mediante un cuestionario que no había diferencias en las características sociodemográficas y económicas de los padres.

En futuras investigaciones y para nuevos programas debería incluirse en el diseño del estudio una valoración de la calidad de la dieta de los escolares antes y después de la intervención. Igualmente sería recomendable incluir en el estudio la valoración del impacto del programa de promoción de la alimentación en el estado de salud nutricional del alumnado, mediante el análisis de sus características antropométricas en comparación con la calidad de su dieta. También es posible que para algunas características no se haya detectado diferencias estadísticamente significativas por limitaciones derivadas del tamaño muestral final. En próximos estudios se deberá contar con una muestra mayor y sería interesante incluir a los tutores/as de los escolares para confirmar las respuestas y determinar si son similares sus hábitos alimentarios a los de sus progenitores.

\section{Conclusiones}

En consecuencia, la puesta en marcha de programas alternativos de promoción de la alimentación saludable puede contribuir a crear hábitos saludables en los niños/as y jóvenes, favoreciendo una mayor adherencia a la DM.

La investigación también ha puesto de manifiesto que los programas de intervención se deberían planificar y evaluar teniendo en cuenta el sexo de los escolares, ya que existen diferencias en el modo en que hombres y mujeres reciben las intervenciones de educación y promoción de la alimentación y en los resultados que se obtienen.

Sería recomendable planificar estos programas dentro de la formación del alumnado a lo largo de su educación escolar obligatoria ${ }^{29}$, pero también en estudios superiores; es más, dada la trascendente relación entre la dieta y el estado de salud, cabría plantearse la posibilidad de que pudieran incluirse materias de alimentación, dietética y nutrición humana en los currículos edu- 
cativos, incluidos los universitarios, tal y como queda recogida en la iniciativa aprobada por el Parlamento Europeo en marzo de 2014, sobre El Patrimonio Gastronómico Europeo: Aspectos Culturales y Educativos.

Es preciso valorar positivamente el compromiso de las instituciones escolares y de las empre- sas de catering con la salud alimentaria de niños y niñas. En la investigación se ha percibido que los centros escolares se preocupan por la educación global de los niños/as, incluyendo en sus proyectos escolares, no sólo lo indicado en el currículo educativo, sino todas las actividades que formen a personas con la mayor calidad de vida posible.

\section{Colaboradores}

Los autores han participado en el diseño del método, análisis de la información, obtención de resultados y tablas, en la generación de conclusiones y discusión, y trasmiten su conformidad a los criterios de autoría de todos los firmantes y su filiación profesional. 


\section{Referencias}

1. Macías AI, Gordillo LG, Camacho EJ. Hábitos alimentarios de niños en edad escolar y el papel de la educación para la salud. Rev Chil Nutr 2012;39(3):40-43.

2. Pérez C, Aranceta J. La dieta mediterránea en el marco de la nutrición comunitaria: luces y sombras. En: ¿Es posible la Dieta Mediterránea en el siglo XXI?. Madrid: IMC; 2011. p. 147-162.

3. Peñalvo JL, Oliva B, Sotos-Prieto M, Uzhova I, Moreno-Franco B, León-Latre M, Ordovás JM. La mayor adherencia a un patrón de dieta mediterránea se asocia a una mejora del perfil lipídico plasmático: la cohorte del Aragon Health Workers Study. Rev Esp Cardiol 2015;68(4):290-297.

4. Martínez MI, Hernández MD, Ojeda M, Mena R, Alegre A, Alfonso JL. Desarrollo de un programa de educación nutricional y valoración del cambio de hábitos alimentarios saludables en una población de estudiantes de Enseñanza Secundaria Obligatoria. Nutr Hosp 2009;24(4):504-510.

5. Contreras J. Vivir en el Mediterráneo antes y ahora. En: ¿Es posible la Dieta Mediterránea en el siglo XXI? 2011. Madrid: IMC; 2011. p. 133-145.

6. Díaz J. Childhood obesity: Prevention or treatment? An Pediatr (Barc) 2017;86 (4):173-175.

7. Ballesteros JM. AESAN y el documento de consenso sobre los comedores escolares. Modelos de programas de comedores escolares en España. Libro Alimentación institucional y de ocio en el siglo XXI: entorno escolar. Madrid [Libro en internet]. 2012 [accedido 2015 Oct 30];4(1):[cerca de 17 p.]. Disponible en: http://www. institutotomaspascualsanz.com/descargas/formacion/publi/Libro_AlimentacionInstitucional_ESCOLAR.pdf

8. Aranceta J, Pérez C, Dalmau J, Gil A, Lama R, Martin MA, Martínez V, Pavón P, Suarez L. El comedor escolar: situación actual y guía de recomendaciones. An Pediatr (Barc) 2008;69(1):72-88.

9. Berradre-Sáenz B, Royo-Bordonada MA, Bosqueda MJ, Moya MA López L. Menú escolar de los centros de enseñanza secundaria de Madrid: conocimiento y cumplimiento de las recomendaciones del Sistema Nacional de Salud. Gac Sanit 2015;29(5):341-346.

10. Soler C. Soberanía alimentaria en las mesas del colegio [Internet]. Amigos de la Tierra en colaboración con la Rev Soberanía Alimentaria, Biodiversidad y Culturas 2011; [cerca de 82 p.]. Disponible en: http://alimentarelcambio.es/wp-content/uploads/2017/02/Dossier soberania_alimentaria_en_el_cole.pdf

11. World Health Organization (WHO). Food and nutrition policy for schools: a tool for the development of school nutritions programs in the European Region. Copenhagen: WHO Regional Office for Europe; 2006.

12. World Health Organization (WHO). School policy framework: implementation of the WHO Global Strategy on diet, physical activity and health. Geneva: WHO; 2008 .

13. González M. La importancia de la agricultura ecológica para el medio ambiente y la salud. Rev Ambiente 2011;95:10-25
14. Barrón, A Muñoz, JM. Los huertos escolares comunitarios: fraguando espacios socioeducativos en y para la sostenibilidad. Foro de educación 2015;13(19):213239.

15. Serra L, Ribas L, Ngo J, Ortega RM, Pérez C, Aranceta J. Alimentación, jóvenes y dieta mediterránea en España. Desarrollo del KIDMED, índice de calidad de la dieta mediterránea en la infancia y la adolescencia. En: Serra Majem L, Aranceta Bartrina J, editores. Alimentación infantil y juvenil. Barcelona: Masson; 2004. p. 51-59.

16. Agencia Española de Seguridad Alimentaria. Estrategia NAOS, Invertir la tendencia de la obesidad. Estrategia para la nutrición, actividad física y prevención de la obesidad. Ministerio de Sanidad y Consumo. Madrid: Ministerio de Sanidad y Consumo; 2005.

17. Gómez-Santos SF, Estévez-Santiago R, Palacios-Gil -Antuñano N, Leis Trabazo MR, Tojo Sierra R, Cuadrado Vives C, Beltrán de Miguel B, Ávila Torres JM, Varela Moreiras G, Casas Esteve R. THAO-child health programme: community based intervention for healthy lifestyles promotion to children and families: results of a cohort study. Nutr Hosp 2015;32(6):25842587.

18. Bibiloni MM, Fernández-Blanco J, Pujol-Plana N, Martín-Galindo N, Fernández-Vallejo MM, RocaDomingo M, Chamorro-Medina J, Tur JA. Mejora de la calidad de la dieta y del estado nutricional en población infantil mediante un programa innovador de educación nutricional: INFADIMED. Gac Sanit 2017;31(6):472-477.

19. Calatayud F, Calatayud B, Gallego JG. Efectos de una dieta mediterránea tradicional en niños con sobrepeso y obesidad tras un año de intervención. Rev Pediatr Aten Primaria 2011;13:553-569.

20. Navarro M, González R, Miguel J. Estudio del estado nutricional de estudiantes de educación primaria y secundaria de la provincia de Valencia y su relación con la adherencia a la Dieta Mediterránea. Rev Esp Nutr Hum Diet 2014;18(2):81-88.

21. Edo A, Montaner I, Bosch A, Casademont MR, Fábrega MT, Fernández A, Gamero M, Ollero MA. Estilos de vida, hábitos dietéticos y prevalencia del sobrepeso y la obesidad en una población infantil. Rev Pediatr Aten Primaria 2010;12(45):53-65.

22. De La Montaña J, Castro L, Cobas N, Rodríguez M, Mínguez M. Adherencia a la dieta mediterránea y su relación con el índice de masa corporal en universitarios de Galicia. Rev Nutr Clín Hosp 2012;32(3):72-80.

23. Rodríguez M, García A, Salinero JJ, Pérez B, Sánchez JJ, García R, Robledo S y Ibañez R. Calidad de la dieta y su relación con el IMC y el sexo en adolescentes. Nutr Clín Diet Hosp 2012;32(2):21-27.

24. Ayechu A, Durá T. Calidad de los hábitos alimentarios (adherencia a la dieta mediterránea) en los alumnos de educación secundaria obligatoria. An Sist Sanit $\mathrm{Na}$ var 2009;3(1):35-42.

25. López E, Navarro M, Ojeda R, Brito E, Ruiz JA, Navarro $\mathrm{M}$. Adecuación a la dieta mediterránea y actividad física en adolescentes de Canarias. Arch Med Deporte 2013;30(4):208-214. 
26. Durá T, Castroviejo A. Adherencia a la dieta mediterránea en la población universitaria. Nutr Hosp 2011;26(3):602-608.

27. Zapico AG, Blández J, Fernández E. Sobrepeso, obesidad y adecuación a la dieta Mediterránea en adolescentes de la Comunidad de Madrid. AMD 2010;XXVII(138):271-280.

28. San Mauro I, Garicano E, González M, Villacorta P, Megías A, Miralles B, Figueroa M, Andrés N, Bonilla MA, Arraz P, Bernal MD, Ruiz AM, Moraleda E, De la Calle L. Hábitos alimentarios y psicológicos en personas que realizan ejercicio físico. Nutr Hosp 2014;30(6):1324-1332.

29. Martínez A, Trescastro EM. Actividades de educación alimentaria y nutricional en escolares de $3^{\circ}$ de primaria en el Colegio Público "La Serranica" de Aspe (Alicante): Experiencia piloto. Rev Esp Nutr Hum Diet 2016;20(2):115-121.

Artigo apresentado em 05/09/2017

Aprovado em 04/05/2018

Versão final apresentada em 06/05/2018 